Discussion There is a significant focus on unmet need for contraception among women in low income countries within the published literature, leaving considerable evidence gaps in relation to unmet need within sexual health generally and among men in particular, and unmet reproductive health need in high income settings. Additionally, using an increased range of data collection methods, analyses and definitions of unmet need would enable better understanding of health inequality in this area.

\section{P281 EXPLAINING EXPERIENCES OF ACCELERATED PARTNER THERAPY PARTNER NOTIFICATION FOR PEOPLE WITH CHLAMYDIA IN THE LUSTRUM RANDOMISED CONTROL TRIAL: PROCESS EVALUATION}

\begin{abstract}
${ }^{1} \mathrm{~F}$ Mapp*, ${ }^{2,3} \mathrm{C}$ Estcourt, ${ }^{4} \mathrm{~J}$ Cassell, ${ }^{2} \mathrm{~J}$ MacQueen, ${ }^{1} \mathrm{~A}$ Howarth, ${ }^{5} \mathrm{~S}$ Brice, ${ }^{5} \mathrm{~A}$ Comer, ${ }^{6} \mathrm{M}$ Symonds, ${ }^{3,7,8} \mathrm{R}$ Nandwani, ${ }^{1} \mathrm{M}$ Woode Owusu, ${ }^{1,9} \mathrm{~J}$ Saunders, ${ }^{1} \mathrm{C}$ Mercer, ${ }^{1} \mathrm{O}$ Stirrup, ${ }^{1} \mathrm{~A}$ Copas, ${ }^{10} \mathrm{~N}$ Low, ${ }^{11} \mathrm{~T}$ Roberts, ${ }^{2} \mathrm{M}$ Pothoulaki, ${ }^{1} \mathrm{~A}$ Tostevin, ${ }^{10} \mathrm{C}$ Althaus, ${ }^{11} \mathrm{C}$ Ogwulu, ${ }^{1,12}$ S Wayal, ${ }^{1} \mathrm{~A}$ Johnson, ${ }^{13} \mathrm{P}$ Flowers. ${ }^{1}$ University College London, London, UK; ${ }^{2}$ Glasgow Caledonian University, Glasgow, UK; ${ }^{3}$ NHS Greater Glasgow and Clyde, Glasgow, UK; ${ }^{4}$ Brighton and Sussex Medical School, Brighton, UK; ${ }^{5}$ Barts Health NHS Trust, London, UK; ${ }^{6}$ Western Sussex Hospitals NHS Foundation Trust, Worthing, UK; ${ }^{7}$ University of Glasgow, Glasgow, UK; ${ }^{8}$ Public Health Scotland, Edinburgh, UK; ${ }^{9}$ Public Health England, London, UK; ${ }^{10}$ University of Bern, Bern, Switzerland; ${ }^{11}$ University of Birmingham, Birmingham, UK; ${ }^{12}$ Development Media International CIC, London, UK; ${ }^{13}$ University of Strathclyde, Glasgow, UK
\end{abstract}

\subsection{6/sextrans-2021-sti.348}

Background Accelerated partner therapy (APT) is a partner notification (PN) method whereby healthcare professionals assess sex partners by telephone, then send or give the index patient antibiotics and self-sampling kits for their sex partner (s). APT was implemented within a cluster cross-over randomised control trial in 17 sexual health clinics in Britain (2018-2019, ISRCTN Reference 15996256). We conducted an integral process evaluation to help explain experiences of using APT.

Methods Focus groups and telephone interviews with 34 healthcare professionals who delivered APT, and telephone interviews with 15 index patients and 17 sex partners who chose APT. Topic guides focussed on how APT was implemented and overall APT experiences. Data were analysed deductively using a bespoke framework derived from initial conceptualisations of APT, and key trial findings.

Results Low uptake of APT was largely because index patients felt it was only suitable for certain types of sex partner. APT was considered best suited to established relationships and not appropriate for relationships with lower emotional connection. However, APT was not always offered by healthcare professionals and many sex partners attended clinic with index patients when they attended for treatment. Nevertheless, those who chose APT felt it worked better than existing options and helped partners overcome barriers to face-to-face care. Most sex partners received APT packs directly from the index patient within a day of consultation; some prioritised taking treatment over self-sampling. Some sex partners reported difficulties in blood sampling (finger-prick) resulting in fewer HIV and syphilis samples being returned than chlamydia and gonorrhoea (urine/vulvo-vaginal swab). Some sex partners did not value testing for infections other than chlamydia/gonorrhoea. Conclusions APT benefits established sexual partnerships with greater emotional connection, by providing treatment rapidly and overcoming barriers to face-to-face care. Targeting of APT combined with interventions to increase sex partner return of self-samples are needed.

\section{P283 DIFFERENCES IN RISK PERCEPTION AND RISK BEHAVIOR BETWEEN PREP-USING AND NON-PREP- USING MSM IN THE NETHERLANDS}

\begin{abstract}
${ }^{1} \mathrm{~S}$ Diexer ${ }^{*},{ }^{1} \mathrm{~A}$ Teslya, ${ }^{2,3} \mathrm{D}$ Rojas Castro, ${ }^{2} \mathrm{R}$ Delabre, ${ }^{4} \mathrm{~K}$ Jonas, ${ }^{1} \mathrm{M}$ Kretzschmar. ${ }^{1} J u l i u s$ Center for Health Sciences and Primary Care, University Medical Center Utrecht, Utrecht University, Utrecht, The Netherlands; ${ }^{2}$ Community-Based Research Laboratory, Coalition PLUS, Pantin, France; ${ }^{3}$ Aix Marseille Univ, INSERM, IRD, SESSTIM, Sciences Economiques and Sociales de la Santé and Traitement de I'IInformation Médicale, Marseille, France; ${ }^{4}$ Department of Work and Social Psychology, Faculty of Psychology and Neuroscience, Maastricht University, Maastricht, The Netherlands
\end{abstract}

\subsection{6/sextrans-2021-sti.349}

Background Pre-exposure prophylaxis (PrEP) uptake has remained low among men who have sex with men (MSM) eligible for PrEP in the Netherlands, which could partly be due to stigma associated with taking PrEP. Perceptions about PrEP users (e.g., PrEP users have an increased rate of (condomless) sexual encounters) may modify mixing behavior and subsequently affect dynamics of HIV and STIs. We investigated whether HIV and STI risk perception, risk behavior, and partner selection depend on PrEP usage.

Methods We analyzed sexual behavior and PrEP data from 426 HIV-negative MSM who participated in the Flash! PrEP in Europe survey in the Netherlands in 2016. We distinguished between current PrEP users $(n=29)$, men interested in $\operatorname{PrEP}(n=267)$, and men not interested in PrEP $(n=130)$. We tested differences between these 3 groups in risk perception and sexual behavior variables using ANOVA and Chi-squared test.

Results Mean age of participants was 42 yrs (range $=18-75$ ); $85 \%$ were born in the Netherlands. PrEP users and men not interested in PrEP consider their risk of acquiring HIV low, while men interested in PrEP consider their risk higher $(p<0.001)$. Perceived risk of getting infected with STIs is higher for PrEP users and men interested in PrEP $(p<0.001)$. PrEP users have significantly more sex partners (mean $=53$, $(\mathrm{SD}=95)$ vs. $13(20)$ vs. 3 (6), p<0.001), and more condomless sex $(\mathrm{p}<0.001)$. PrEP users and men interested in PrEP are less likely to refuse to have sex and more likely to have condomless sex with a PrEP user $(\mathrm{p}<0.001)$.

Conclusion PrEP users engage in sexual behavior with higher risk of STI acquisition than non-PrEP users. Men not interested in PrEP are less open for sexual encounters with PrEP users than men in other groups. Therefore, perceptions of PrEP users and non-PrEP users may change mixing patterns and thus STI dynamics.

\section{P286 QUALITATIVE FINDINGS FROM NATSAL-COVID: EXPLORING DIFFICULTIES AND DISTRESS WITHIN ESTABLISHED RELATIONSHIPS DURING COVID-19 PANDEMIC}

${ }^{1} \mathrm{R}$ Boso Perez ${ }^{*},{ }^{1} \mathrm{~K}$ Maxwell, ${ }^{2} \mathrm{D}$ Reid, ${ }^{3} \mathrm{C}$ Tanton, ${ }^{3} \mathrm{~W}$ Macdowall, ${ }^{3} \mathrm{C}$ Bonell, ${ }^{2} \mathrm{~S} C$ Clifton, ${ }^{2} \mathrm{P}$ Sonnenberg, ${ }^{2} \mathrm{C}$ Mercer, ${ }^{2} \mathrm{~N}$ Field, ${ }^{1} \mathrm{~K}$ Mitchell. ${ }^{1}$ University of Glasgow, Glasgow, UK; ${ }^{2}$ University College London, London, UK; ${ }^{3}$ London School of Hygiene and Tropical Medicine, London, UK

10.1136/sextrans-2021-sti.350 